\title{
Psychological targeting: nudge or boost to foster mindful and sustainable consumption?
}

\author{
Erik Hermann ${ }^{1}$
}

Received: 21 June 2021 / Accepted: 8 February 2022

(c) The Author(s) 2022

Marketing activities fuel consumption, which can eventually harm consumer, social, and environmental well-being. Excessive increases in consumption undermine the transition to sustainability, leading to calls for sufficiency-oriented lifestyles as well as for mindful and sustainable consumption. In this context, artificial intelligence (AI) has to be a driving force in reconciling the seemingly incompatible goals of marketing (e.g., sales and consumption growth) on one hand and mindful and sustainable consumption on the other hand. The massive amount of available consumer data (i.e., digital records like search queries or social media data) allow an unprecedented understanding of consumers' wants and needs. They can be even used as a window into consumers' psychology. By AI-driven prediction of consumers' psychological traits and states from their digital footprints and respective tailoring of communication messages and interventions, consumers that are (psychologically) predisposed and responsive to mindful and sustainable can be identified and targeted (i.e., psychological targeting). Particularly, the sophistication and computational power of AI applications in combination with Big Data can account for individual and psychological factors (e.g., personality, values, intelligence etc.) that constitute barriers to or drivers for consumers' mindful and sustainable consumption intentions and behavior. Conversely, consumers who are prone to addictive and compulsive behaviors or compulsive buying could be targeted by messages or interventions mitigating these adverse tendencies, for instance, by emphasizing the individual or environmental consequences of excessive or compulsive buying. Thus, data- and AI-driven marketing has to be leveraged for shifting consumers to more mindful and sustainable consumption behavior. Thereby, AI in marketing provides a dual advantage for society at large by

\section{Erik Hermann}

hermann@ihp-microelectronics.com

1 Wireless Systems, IHP - Leibniz-Institut für innovative Mikroelektronik, Frankfurt (Oder), Germany promoting societal and environmental well-being while preventing harm for consumers.

\section{Ethical concerns of Al-driven psychological targeting}

The pervasiveness of AI systems, applications, and devices has inflamed the debate of ethical principles and values guiding AI development and use. In light of AI's impact on the individual, economic, and societal level, the AI ethics literature increasingly focuses ethical frameworks for AI for (social) good, which comprise the principles beneficence, non-maleficence, autonomy, justice, and explicability (Floridi et al. 2018). While beneficence refers to the promotion of individual, social, and environmental well-being (e.g., sustainability, mindfulness), non-maleficence entails the prevention of risks and any harm (e.g., overconsumption, addictive and compulsive consumption). Autonomy advocates self-determination and balancing human and AI agency, whereas justice refers to fairness and the avoidance of unwanted/unfair biases. Finally, explicability covers intelligibility (i.e., how AI works) and accountability (i.e., who is responsible for the way AI works). The most striking ethical concerns related to AI-driven psychological targeting arise in respect to privacy and data protection (i.e., non-maleficence), consumer autonomy, unbiased and antidiscriminatory outcomes (i.e., justice), and the basic understanding of AI applications' functioning (i.e., intelligibility). First, the sensitive consumer data feeding AI-based psychological targeting applications can substantially interfere with privacy and data protection. Second, consumer should be able to decide themselves whether and how to engage with AI-powered psychologically tailored messages to facilitate consumer autonomy. Third, psychologically personalized messages and interventions should not target or manipulate vulnerable groups, exploit individuals' weaknesses, or discriminate certain consumer groups against others on the 
basis of psychological traits and states and individual factors. Finally, consumers need a basic understanding of how AI works to assess whether and how AI systems (positively or negatively) shape their decision-making.

\section{Nudge or boost for good?}

Psychologically tailored messages and interventions can take the form of nudges or boosts (Hertwig and Grüne-Yanoff 2017). While nudges shape and alter the consumer decision environment (i.e., which choices and related information consumers encounter), boosts are interventions that foster consumers' cognitive and motivational competences (e.g., through changes in skills or knowledge). They do not just present pertinent and accurate information or influence immediate behavior by changing the options consumers are exposed to, but they aim at empowering individuals to make better decisions according to their own goals and preferences (Hertwig and Grüne-Yanoff 2017). To promote more beneficial and less adverse consumer decision-making in the long run, consumer-literacy-oriented boosts have to be preferred over nudges. Moreover, to account for consumer agency (i.e., autonomy) and privacy (i.e., non-maleficence), boosts have to be accompanied by informed consent and an opt-in model. Thereby, boosts are transparent by design and require consumers' active cooperation. AI-driven, psychologically targeted boosts would then attempt to empower consumers to self-monitor and scrutinize their consumption behavior and patterns in respect to sustainable and mindful purchase decisions. In other words, they aspire to strengthen consumers' awareness and literacy in relation to environmentally relevant product attributes and mindful and sustainable consumption in general. Following a learning rationale, data about consumer decisions feed AI systems and applications to optimize boosts and achieve a virtuous cycle of self-empowerment.

In conclusion, sustainable development and consumption is an imperative to facilitate future individual, social, and environmental well-being. AI-driven psychologically targeting can be a valuable approach to foster mindful and sustainable consumption. Thereby, consumers should be empowered by AI-driven, psychologically targeted and tailored boosts to promote responsible and conscious decision-making. Public policy can likewise benefit from the insights generated to establish policies and interventions pursuing durable mindful and sustainable consumption patterns on a societal level. To fully leverage AI for social good and consumer empowerment, consumer autonomy and privacy have to be particularly taken into account for the development, design, and deployment of AI-enabled boosts.

Curmudgeon Corner Curmudgeon Corner is a short opinionated column on trends in technology, arts, science and society, commenting on issues of concern to the research community and wider society. Whilst the drive for super-human intelligence promotes potential benefits to wider society, it also raises deep concerns of existential risk, thereby highlighting the need for an ongoing conversation between technology and society. At the core of Curmudgeon concern is the question: What is it to be human in the age of the AI machine? -Editor.

Funding Open Access funding enabled and organized by Projekt DEAL.

Open Access This article is licensed under a Creative Commons Attribution 4.0 International License, which permits use, sharing, adaptation, distribution and reproduction in any medium or format, as long as you give appropriate credit to the original author(s) and the source, provide a link to the Creative Commons licence, and indicate if changes were made. The images or other third party material in this article are included in the article's Creative Commons licence, unless indicated otherwise in a credit line to the material. If material is not included in the article's Creative Commons licence and your intended use is not permitted by statutory regulation or exceeds the permitted use, you will need to obtain permission directly from the copyright holder. To view a copy of this licence, visit http://creativecommons.org/licenses/by/4.0/.

\section{References}

Floridi L, Cowls J, Beltrametti M, Chatila R, Chazerand P, Dignum V et al (2018) AI4People-an ethical framework for a good AI society: opportunities, risks, principles, and recommendations. Minds Mach 28:689-707. https://doi.org/10.1007/s11023-018-9482-5

Hertwig R, Grüne-Yanoff T (2017) Nudging and boosting: Steering or empowering good decisions. Perspect Psychol Sci 12:973-986. https://doi.org/10.1177/1745691617702496

Publisher's Note Springer Nature remains neutral with regard to jurisdictional claims in published maps and institutional affiliations. 